\title{
Role of self-based and sample-based consensus estimates as mediators of responsibility judgments for automobile accidents
}

\author{
SHIGERU HAGIWARA \\ Department of Psychology, Faculty of Letters, Chiba University, Chiba 260
}

\begin{abstract}
In order to explain the process of responsibility judgments for an automobile accident, the estimates of self-based consensus (i.e., would I have behaved as the motorist did?) and samplebased consensus (i.e., would ordinary people have behaved as the motorist did?) were introduced as mediational variables. As expected, for all three accident situations utilized in the present study, the extent of responsibility attributed to the motorist was found to be negatively correlated with both self-based and sample-based consensus estimates, and a highly positive correlation was observed between these two types of consensus estimates. In addition, a consistent tendency of differences emerged when subjects were divided into two groups on the basis of whether or not they had a driver's license, in that driver subjects generated lower consensus estimates and attributed greater responsibility to the accident perpetrator than did nondriver subjects. Implications of these results for the issue of self-protective motivational biases were discussed, together with the conceptual issue of responsibility which was addressed to in the present study by incorporation of test-retest procedure and by inclusion of sanction measures.
\end{abstract}

Key words: responsibility, defensive attribution, self-based consensus, sample-based consensus, false consensus bias.

Since Walster's intriguing study in 1966 , the assessment of responsibility attributed for an accidental occurrence has become the subject of considerable research interest, and much of the effort in this line is directed to demonstrate self-protective motivational biases proposed by Walster and later reformulated by Shaver (1970a) as "defensive attribution". Unfortunately, the results of these studies have been quite inconsistent (see Burger, 1981, for a review), and invited methodological and conceptual critiques (Fishbein \& Ajzen, 1973; Vidmar \& Crinklaw, 1974).

Walster originally postulated that the need to assign responsibility on the part of perceivers to someone potentially at fault, not to chance, increases as consequences of an accident become more serious, because for perceivers to attribute a catastrophe to chance has the threatening implications that an accident of equal magnitude could capriciously happen to them also. In support of this postulation she found a positive association between responsibility attribution and accident severity in a study which utilized an automobile accident case with varying outcome severities.

Later studies including her own, however, often failed to replicate this severityresponsibility relation (e.g., McKillip \& Posavac, 1975; Schroeder \& Linder, 1976; Shaver, 1970a, 1970b; Walster, 1967). Among 22 relevant studies Burger cited for the purpose of a meta-analytic statistical procedure he found only six which supported the proposed relation, although his overall conclusion was that, all the results combined, there is a statistically significant but weak tendency to attribute more responsibility to an accident perpetrator for a severe as opposed to a mild accident.

In order to account for the failure to replicate the finding of increased assignment of responsibility for serious outcomes, Shaver proposed the " blame-avoidance", rather than the "harm-avoidance" as suggested by Walster, attributional tend- 
ency by introducing concepts of situational and personal relevance. That is, when a particular type of accident is situationally relevant and an accident perpetrator personally similar to perceivers, they tend to attribute lessened responsibility for a severe accident lest they be blamed should they cause a similar accident. Here situational relevance, as well as certain degree of outcome severity, is regarded as a prerequisite for the arousal of self-protective attribution of responsibility; and, once the self-protective motivation is established, further increase in relevance due to personal similarity is thought to make the tendency of "blameavoidance" surpass that of "harm-avoidance", thus resulting in decreased responsibility attribution for an accident perpetrator.

This interactive effect of personal similarity has fared much better in empirical testings than the simple severity-dependent effect. Given that the situation of an accident is relevant and its outcome severe, the tendency of perceivers to attribute less responsibility to personally similar than to dissimilar accident perpetrators has been repeatedly demonstrated (Chaikin \& Darley, 1973; McKillip \& Posavac, 1975; Shaver, 1970a; Shaw \& McMartin, 1977).

By extending this notion of defensive attribution to an automobile accident situation, it may be expected that motorists would be more lenient in their judgment and attribute lowered responsibility to someone involved in an accident, a possibility also suggested by Lloyd-Bostock (1981, p. 156). The opposite tendency emerged, however, in our previous study which investigated responsibility judgments of undergraduate students regarding six different cases of automobile accidents (Hagiwara, Sono, \& Sano, 1977). Dividing the subjects into drivers and nondrivers on the basis of whether they had a driver's license or not, we unexpectedly found a general tendency of the former to attribute greater responsibility to an accident perpetrator (a motorist) than the latter. Therefore, one purpose of the present study is to replicate the difference found between driver and nondriver subjects in their responsibility attribution for automobile accidents, and also to account for the difference by introducing some mediational variables affecting responsibility judgments.

In his pioneering work on attribution processes Heider (1958) stated that the person may not be held responsible if " anybody would have felt and acted as he did under the circumstances" ( $p, 114)$. Translating into Kelley's (1967) covariation principle, it can be restated that perceived high consensus regarding the target behavior in a given situation would lead to lowered responsibility attributed to the agent.

Even though the explicit consensus information is not usually provided in responsibility attribution research for an accident, a perceiver may generate such a sample-based consensus estimate in the process of judging responsibility, i.e., how other people would react to the situation in which the agent was placed. Furthermore, it may be possible to assume that such a sample-based consensus estimate is mediated by a perceiver's self-based consensus estimate, i.e., how I would react to the same situation. In this regard Ross (1977) used the term "false consensus bias" to designate the tendency of people to "see their own behavioral choices and judgments as relatively common and appropriate while viewing alternative responses as uncommon, deviant, and inappropriate" (p. 188). Therefore, it is predicted that, in proportion as perceivers feel that they themselves would react as the agent did to the situation, they tend to see the agent's behavior as more common and increasingly estimate that other people would react similarly. These consensus estimates, in turn, are expected to affect responsibility attribution. The 
more the target behavior in a given situation is seen as common, the less responsibility would be attributed to the agent.

Thus, it is postulated that a perceiver's self-based consensus estimate would affect responsibility attribution through its generalization to the sample-based consensus estimate. In addition to this indirect effect of the self-based consensus estimate on responsibility attribution, its direct effect may be considered. That is, the estimate of self-based consensus may affect responsibility judgments independently of its generalization to sample-based consensus. In a study which independently manipulated self-based and sample-based consensus Hansen and Donoghue (1977, Experiment 3) reported the greater efficacy of self-based consensus as determinants of causal attributions.

These mediational variables of consensus estimates could, then, be applied to account for the driver-nondriver difference in responsibility attribution for an automobile accident. It may be that, relative to subjects who do not drive, driver subjects estimate lower self-based consensus in judging responsibility for the person involved in an automobile accident. Let alone whether this is based on self-protective motivation or rational calculus derived from their own driving experiences, the perception of lower selfbased consensus, together with its generalization to lower sample-based consensus, would lead to greater responsibility attributed to the accident perpetrator.

In summary, two mediational variables of self-based and sample-based consensus estimates were introduced in the present study to explain the process of responsibility attribution. It is hypothesized that, in judging responsibility of the agent, a perceiver generates a self-based consensus estimate which, in combination with its generalization to a sample-based consensus estimate, determines the extent of responsibility attributed to the agent. More specifically, self-based consensus esti- mates are predicted to be positively correlated with sample-based consensus estimates, and these self-generated consensus estimates, in turn, are predicted to be negatively correlated with the extent of responsibility attributed. Accordingly, the difference between drivers and nondrivers in their responsibility attribution for automobile accidents was held to be explicable by their different estimates of self-based consensus regarding the target behavior of the motorist involved in an accident. In other words, greater responsibility attribution of driver subjects was assumed to be mediated by their lower self-based consensus estimates.

Additionally, in relation to the conceptual issue of responsibility, reliability of responsibility judgments as well as other judgments including consensus estimates was assessed in the present study. Even though responsibility judgments are suggested to be very intricate and sensitive to the situation in which they are elicited, no studies have so far addressed their attention to the issue of reliability of such judgments over repeated measurements separated in time. Also in order to examine the generalizability of the findings, three different stimulus stories were employed. Thus, the subjects including both drivers and nondrivers were twice requested to respond to three stimulus stories, each depicting a different automobile accident situation.

\section{Method}

\section{Procedure}

A booklet which contained three stimulus stories of automobile accidents accompanied by dependent measures was administered to undergraduate students in their college classes. Following the instruction printed on the cover sheet, subjects proceeded to read each stimulus story and responded to dependent measures. Some personal information such as name, sex, age of subjects and whether or not 
they had a driver's license was requested on the last page of the booklet. This whole procedure was repeated eight weeks later to assess the test-retest reliability of each dependent measure, and only the data from those subjects who responded twice were analyzed.

\section{Subjects}

The subjects were undergraduate students attending two major universities in Tokyo and Chiba, who were recruited and tested in their regular class meetings. The total number of participants was 235 for the first session and 195 for the second (retest) session, but only the data from those attending both sessions, which amounted to 186 subjects, were used for analysis. Of this total of 186 subjects, 80 were males and 106 were females, and the male sample included 43 drivers and 37 nondrivers while the female sample had 28 drivers and 78 nondrivers.

\section{Stimulus Materials}

Three vignettes, each depicting a different situation of automobile accidents, were used as stimulus materials. Of these three materials, two were adapted from the actual cases tried in court (Case 1 and 3) and one was hypothetical (Case 2). Even though the accident situations depicted were different for each case, they had some features in common in their structure. First, unlike some versions of Walster's (1966) accident situation in which only a perpetrator suffered, a perpetrator and a victim were separated in the present cases where a motorist always harmed a person who was not driving. Second, all the accident situations were so constructed as to inject a certain degree of conflict in judging responsibility in the sense that it is possible to find fault with both sides, motorist and victim. Third, the consequence in all the accident situations was similar in that the victim always suffered an injury which would take a week to heal.
Let us here cite one stimulus story (Case 3) for illustration:

Around 3 p.m. on December 11 a seven-year-old first grader Nobuko Sawada was hit by a car while she was playing on the street ( 4.5 meters in width) in front of her house, suffering an injury on her right ankle which would take a week to heal. A police examination later found out the following: Having put herself in an empty cardboard box $(95 \mathrm{~cm} \times 78 \mathrm{~cm} \times 65 \mathrm{~cm}$ in size) which used to contain a washing machine the Sawadas had bought a day before, Nobuko was at that time trying to roll it over for fun. A 30 . year-old company employee Akihiko Hayashida, while driving his car, noticed the box about 60 meters ahead rolling over a few times toward the center of the street. Thinking it was an empty box being blown by the wind, he continued to proceed at the speed of 25 kilometers per hour without paying further attention. Thus he hit the box and Nobuko in it with the left front wheel of his car.

This case was adapted from our previous study (Hagiwara et al., 1977) for the reason that, among six different accident situations previously utilized, the drivernondriver difference in responsibility attribution was most clearly pronounced in this stimulus story. The other two stimulus stories were newly devised for the present study. One was about an accident in which a drunkard lying on a dark street at night was hit by a motorist (Case 1) and the other depicted a situation where an intoxicated man who was trying to climb up to the roof of a parked car fell down to the ground because the startled driver of the car suddenly pulled away (Case 2). An illustrative figure was attached to each stimulus story in order to help subjects better comprehend the accident situation depicted. 


\section{Dependent Measures}

Following each stimulus story was a series of three questions to be answered in percentage terms, which constituted the main dependent measures of the present study. The first question asked the extent to which the motorist was responsible for the occurrence of the accident in terms of percentage, $0 \%$ representing "not at all responsible" and 100\% "totally responsible". The next two questions dealt with, respectively, the estimates of sample-based and self-based consensus regarding the motorist's behavior, which were put in the following format:

[sample-based consensus estimate] "Given the situation (driver name) had faced, how do you think ordinary people would react? Supposing 100 motorists randomly picked were placed in the situation, how many of them do you estimate would react as (driver name) did?"

[self-based consensus estimate] "Supposing you were placed in the situation, how would you estimate the probability in percentage terms of your reacting as (driver name) did?"

These main dependent measures were followed by a set of ten statements, to each of which subjects were asked to indicate their degree of endorsement by responding on a 5-point scale ranging from "strongly disagree" (1) to "strongly agree" (5). Two of these statements, common to all cases, were about assignment of sanctions to the perpetrator. One was about restitution, "(driver name) should pay compensation to (victim name) for the injury ", and the other was about punishment, " (driver name) should have his/her driver's license suspended". The rest of the statements were mostly concerned with perception of the particular accident situation. Even though sanction measures such as imposition of monetary fines and/or jail terms were often assumed to be equiva- lent to the notion of responsibility attribution (e.g., McKillip \& Posavac, 1975; Phares \& Wilson, 1972), few studies have reported the correlation between responsibility attribution and sanctioning. ${ }^{1}$ Therefore, the two items concerning the applicability of sanctions were included to illuminate the conceptual issue of responsibility.

Additionally, personality impressions of both the perpetrator and the victim were assessed, respectively, on an identical series of seven bipolar adjective scales with five units placed between each pair of adjectives. To summarize, the dependent measures accompanying each stimulus story consisted of 27 items, i.e., 3 main questions to be answered in percentage terms, 10 Likert-type scales concerning the prescribed statements including the two related to sanctions, and 14 bipolar adjective scales to assess impressions of both the perpetrator and the victim, thus amounting to a total of 81 items for all three cases included.

\section{Results}

\section{Test-retest Reliabilities}

For each of the 81 items the test-retest reliability was calculated as a Pearson's product-moment correlation coefficient for the responses of 186 subjects on the two sessions. Each of these coefficients was then transformed by Fisher's $r$-to- $z$ transformation to obtain the average correlation for all items. The average correlation, transformed back from the Fisher $z$ score, was .510 and indicated a significant degree of stability for 186 subjects responding to the 81 items. By combining these 81 correlations into groupings, the average correlation was calculated in the same manner for each stimulus story and

\footnotetext{
1 McKillip and Posavac (1975, Experiment 1) reported a positive correlation $(r=.46)$ between size of fines and responsibility assigned to the perpetrator of an automobile accident.
} 
for each type of dependent measure. Even though the average correlation was quite constant over different stimulus materials, its value being $.510, .515$, and .507 for Case 1, 2, and 3 respectively, it differed somewhat by item types. The main questions responded in percentage terms showed the greatest consistency of responses over the 8-week interval, followed by the evaluative responses to prescribed statements, and the bipolar adjective scales had the smallest stability. The average correlation for each type of item was $.599, .550$, and .460 in order of its size.

A Pearson's product-moment correlation was also calculated to assess the stability of responses for each of the 186 subjects for the 81 responses on the two identical questionnaires. Each of these coefficients, which ranged from .640 to .999 , was again converted to the $z$ score and mean value over individuals was calculated. The average correlation for all subjects was .921 and indicated a very strong degree of stability of judgments over the 8-week period. Since the responses of the 186 subjects to the 81 items on the first and second sessions were found to be highly correlated, they were pooled over the two sessions to obtain each subject's mean response to each item, which constituted the data for the further analysis. ${ }^{2}$

\section{Intercorrelations of Main Dependent Measures}

On the basis of the mean value of each subject's responses on both sessions, Pearson's product-moment correlations were computed among the dependent measures for each case separately and across cases using the data pooled over the different stimulus stories. Two major correlation patterns are readily noted in Table 1 which summarizes the intercorrelations of three main dependent measures of the

\footnotetext{
2 All the results reported in the present article remained almost identical when the data were analyzed separately for each session.
}

Table 1

Correlations among responsibility attribution $(X)$, sample-based consensus estimate $(Y)$, and self-based consensus estimate (Z)

\begin{tabular}{|c|c|c|c|c|}
\hline & \multicolumn{3}{|c|}{ Case } & \multirow{2}{*}{ Pooled } \\
\hline & 1 & 2 & 3 & \\
\hline$r_{X Y}$ & -.289 & -.277 & -.360 & -.251 \\
\hline$r_{X z}$ & -.351 & -.232 & -.423 & -.306 \\
\hline$r_{1 Z}$ & .694 & .780 & .795 & .711 \\
\hline
\end{tabular}

present study, i.e., responsibility attribution $(\mathrm{X})$, sample-based consensus estimate $(\mathrm{Y})$, and self-based consensus estimate $(\mathrm{Z})$.

First, strong positive correlations emerged between the estimates of sample-based and self-based consensus regarding the perpetrator's behavior, a clear indication of the false consensus bias as suggested by Ross (1977). The subjects tended to see their own behavioral choices as normative, assuming that they were shared by many others. Second, in support of Kelley's covariation principle, these consensus estimates were found to be negatively correlated with the extent of responsibility attributed to the perpetrator. The more the subjects thought that they themselves, therefore others as well, would react as the target motorist did to the situation in which the accident occurred, the less responsibility they attributed to the motorist for the occurrence of the accident.

Thus, the predicted patterns of correlations were confirmed among the main dependent variables for each case analyzed separately and across cases when each subject's responses were pooled over the different accident situations. The robustness of these results was further established as we obtained the same correlation patterns when the data were analyzed separately for drivers and nondrivers, and for each sex of the subjects.

Path Analyses

In addition to the correlation patterns 
of the three main dependent variables, we also postulated their implicit causal relations (see Fig. 1). The present results, of course, do not establish the causal relations of the variables. However, there is subsidiary evidence which supports the assumption that the self-based consensus estimate determines both responsibility attribution and the sample-based consensus estimate, not vice versa. When the test-retest reliability of each item was compared, the self-based consensus estimate showed the highest stability over the 8 -week interval. The correlation of responses on both sessions averaged over the different stimulus stories via Fisher's $r$-to- $z$ transformation was .538 for responsibility attribution, .577 for sample-based consensus estimate, and .670 for self-based consensus estimate. Though not conclusive, if it is assumed that kernel responses are less subject to variation over time, these test-retest reliability results may be regarded as supporting the postulated causal sequences of the three variables.

For this causal model, though it is admittedly too simple to fully account for the process of responsibility judgments, a path analysis was performed to evaluate the relative contributions of self-based and sample-based consensus estimates in determining responsibility attribution. The

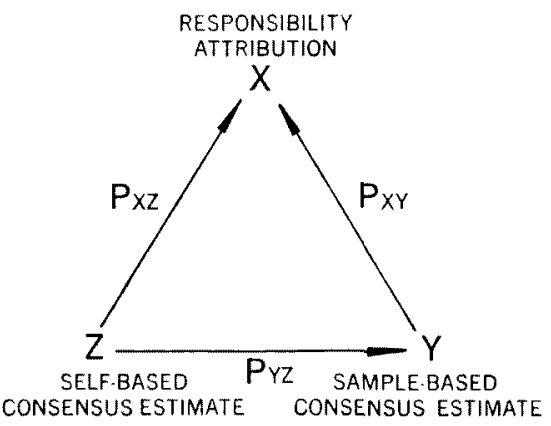

Fig. 1. Postulated causal model of the relationships among responsibility attribution $(\mathrm{X})$, samplebased consensus estimate ( $Y$ ), and self-based consensus estimate (Z).
Table 2

Results of path analyses for the postulated causal model

\begin{tabular}{ccccc}
\hline & \multicolumn{3}{c}{ Case } & Pooled \\
\cline { 2 - 5 } & \multicolumn{1}{c}{1} & \multicolumn{1}{c}{2} & \multicolumn{1}{c}{3} & \\
\hline$p_{\mathrm{X} Y}$ & -.088 & -.245 & -.064 & -.068 \\
$p_{X Z}$ & -.290 & -.041 & -.372 & -.258 \\
$p_{\mathrm{X} Z}$ & .694 & .780 & .795 & .711 \\
\hline$R^{2}$ & .127 & .077 & .180 & .096
\end{tabular}

estimated path coefficients for the respective paths, together with the proportion of variance in responsibility judgments accounted for by the model $\left(R^{2}\right)$, are summarized in Table 2 for each case separately and across cases based on the responses pooled over the different stimulus materials.

As indicated by the different values of the coefficients assigned for the respective paths, the direct effect of the self-based consensus estimate on responsibility attribution was found to be generally greater than that of the sample-based consensus estimate. In fact, excepting Ciase 2, the path coefficient was quite small for the linkage leading from the sample-based consensus estimate to responsibility attribution. It may be that, the self-based consensus estimate being a decisive factor, significant zero-order correlations observed between the sample-based consensus estimate and responsibility attribution were rather spurious for these cases.

\section{Relationship between Responsibility and Sanc- tions}

Additionally, in regard to the conceptual issue of responsibility, the relationship between responsibility attribution (X) and two sanction measures, i.e., restitution to the victim (R) and punishment in terms of license suspension $(P)$, was examined. Pearson's product-moment correlation coefficients computed between these variables are summarized in Table 3 for cach case separately and across 
Table 3

Correlations among responsibility attribution $(X)$, restitution $(R)$, and punishment $(P)$

\begin{tabular}{ccccc}
\hline & \multicolumn{3}{c}{ Case } & Pooled \\
\cline { 2 - 5 } & 1 & 2 & 3 & \\
\hline$r_{\mathrm{XR}}$ & .502 & .482 & .656 & .587 \\
$r_{\mathrm{XP}}$ & .396 & .395 & .542 & .446 \\
$r_{\mathrm{HP}}$ & .376 & .620 & .484 & .507
\end{tabular}

Note: All correlations are significant at $p<.01$.

cases based on the data pooled over the different stimulus stories.

The extent of responsibility attributed was highly correlated with the degree of applicability of both restitution and punishment, with the latter two sanction measures also highly correlated themselves. The subjects who attributed greater responsibility to the target motorist were more likely to think that the motorist should pay compensation to the victim and that the motorist's license should be suspended. Thus, responsibility attribution and assignment of sanctions were found to covary in the present study, though it does not mean that responsibility judgments can be equated with sanctioning nor that responsibility and sanction measures are equally sensitive to the same influencing factors.

\section{Differences between Driver Subjects and Non- driver Subjects}

Depending on whether or not they had a driver's license, the subjects were divided into two groups of drivers and nondrivers, and a one-way analysis of variance was performed to evaluate the difference between the groups for each dependent measure. Mean scores of the respective groups on each of the three main dependent measures and two sanction measures, together with the resultant $F$ ratios pertaining to the group difference, are summarized in Table 4 for each case separately and across cases based on the data averaged over the different stimulus ma- terials. On the whole, the predicted directions of difference between driver and nondriver subjects were confirmed in the present results. However, the difference was not equally pronounced for all stimulus stories nor for all relevant dependent measures.

The driver-nondriver difference was most clearly indicated with respect to their self-based consensus estimates. As expected, the estimated self-based consensus was lower for driver subjects than for nondriver subjects, and the difference was statistically significant for all stimulus stories analyzed separately and combined. Thus, relative to subjects who do not drive, driver subjects estimated the lower probability of their reacting as the target motorist did to the situation in which the accident occurred, thereby implying the lessened likelihood of their causing similar accidents. This may be taken as evidence for the motivational bias of harm-avoidance tendency on the part of driver subjects, although other interpretations are possible.

Regarding the target behavior of the motorist, different self-based consensus estimates of drivers and nondrivers generalized to their sample-based consensus estimates. The estimated sample-based consensus was also lower for driver subjects than for nondriver subjects, and the difference was significant for the pooled data and for two of the three stimulus stories (Case 1 and 3) analyzed separately. In other words, driver subjects generally perceived the motorist's behavior as less likely to be shared by others, therefore less common, than did nondriver subjects. Thus, compared to nondriver subjects, driver subjects tended to think that not only they themselves but other people also would be less likely to react as the perpetrator did to the situation in which the accident occurred.

These differences in consensus estimates between driver and nondriver subjects, in turn, seemed to be reflected in 
Tablc 4

Mean scores of driver subjects and nondriver subjects on measures of responsibility attribution $(X)$, sample-based consensus estimate $(Y)$, self-based consensus estimate ( $\mathrm{Z})$, restitution (R), and punishment $(\mathrm{P})$

\begin{tabular}{|c|c|c|c|c|c|}
\hline Group & $x$ & $Y$ & $Z$ & $\mathrm{R}$ & $\mathrm{P}$ \\
\hline \multicolumn{6}{|c|}{ Case 1} \\
\hline driver & 51.94 & 43.80 & 34.59 & 2.77 & 2.13 \\
\hline nondriver & 48.00 & 50.84 & 43.03 & 2.40 & 1.81 \\
\hline$F$ & 1.73 & $4.62 *$ & $5.33^{*}$ & $5.93^{*}$ & $4.76^{*}$ \\
\hline \multicolumn{6}{|c|}{ Case 2} \\
\hline driver & 35.27 & 50.89 & 41.03 & 1.78 & 1.11 \\
\hline nondriver & 33.21 & 55.42 & 50.97 & 1.64 & 1.18 \\
\hline$F$ & .42 & 1.74 & $5.01 *$ & .91 & .25 \\
\hline \multicolumn{6}{|c|}{ Case 3} \\
\hline driver & 64.82 & 32.58 & 21.68 & 3.15 & 2.47 \\
\hline nondriver & 53.62 & 39.77 & 31.00 & 2.78 & 2.23 \\
\hline$F$ & $16.25^{* *}$ & $5.57^{*}$ & $8.31^{* *}$ & $9.60 * *$ & 3.17 \\
\hline \multicolumn{6}{|c|}{ Pooled } \\
\hline driver & 50.84 & 42.42 & 32.43 & 2.57 & 1.90 \\
\hline nondriver & 44.94 & 48.68 & 41.67 & 2.28 & 1.74 \\
\hline$F$ & $6.61 * *$ & $6.80^{* *}$ & $10.78 * *$ & $6.41 * *$ & 2.04 \\
\hline
\end{tabular}

Note: The number of subjects was 71 for the driver group and 115 for the nondriver group; degrees of freedom for $F$ ratios were 1, 184. Higher scores on $\mathrm{R}$ and $\mathrm{P}$ indicate, respectively, stronger agreements to the statement that the motorist should pay compensation to the victim for the injury and that the motorist should have his/her driver's license suspended.

$* p<.05, * * p<.01$.

their responsibility attributions. It was postulated that lower consensus estimates of driver subjects would result in their greater responsibility attribution for the target motorist. Although the obtained results were consistent with this postulation, the driver-nondriver difference was not as clearly pronounced for their responsibility attribution as for their consensus estimates. When the data were pooled over the different accident situations, the overall responsibility attributed to the motorist was significantly greater for driver subjects than for nondriver subjects. However, when each case was separately analyzed, a significant difference was not obtained for two of the three cases (Case 1 and 2). As to the accident situation adapted from our previous study (Case 3 ) the driver-nondriver difference was highly significant, thus our previous result was replicated.

The parallel direction of difference between driver and nondriver subjects was also observed for their responses to two sanction measures. Contrary to predictions based on the blame-avoidance tendency, driver subjects were found to be generally more strict, not lenient, with the accident perpetrator than were non- 
driver subjects. That is, relative to subjects who do not drive, driver subjects were more willing to think that the perpetrator should pay compensation to the victim and that the perpetrator's license should be suspended. These two sanction measures, however, were not equally sensitive to the same influencing factor. The driver-nondriver difference was more clearly manifested in the restitution measure than in the punishment measure. The overall difference between driver and nondriver subjects, based on the data pooled over the different accident situations, was statistically significant for the former, but not for the latter. Furthermore, when each case was separately analyzed, the difference as to the restitution was significant for two of the three cases (Case 1 and 3), whereas a significant difference was observed only for one stimulus story (Case 1) as to the punishment.

Lastly, the confounding of subjects' sex with the driver-nondriver grouping should be mentioned. Since the proportion of female subjects was much higher for the nondriver group than for the driver group, the differences observed between the two groups may have reflected the different proportion of males to females, not the driver-nondriver difference per se, given that the responses on relevant dependent measures differ by sex of the subjects. Accordingly, a one-way analysis of variance was performed to evaluate the difference between male and female subjects for each dependent measure. Even though a few significant sex differences were found, they did not parallel the differences reported between driver and nondriver subjects. Moreover, the effects of subjects' sex did not interact with the driver-nondriver difference. The directions of difference between drivers and nondrivers were found to remain intact for both males and females, when the data were analyzed separately for each sex.

\section{Discussion}

On the whole, the present results supported the efficacy of the predicted role of self-based and sample-based consensus estimates as mediators of responsibility judgments. First of all, in line with the prediction that perceived high consensus would lead to lowered responsibility attribution, for all accident situations the extent of responsibility attributed to the perpetrator was found to be negatively correlated with the estimates of both selfbased and sample-based consensus regarding the perpetrator's behavior in question. Some caution is needed, however, in generalizing their effect to other situations of responsibility judgments, because the mediational role of consensus estimates is probably limited to the cases where a perceiver can conceive of some possibility of sharing the target person's behavior in a given situation. For criminal acts such as rape, for example, responsibility judgments are not likely to be mediated by consensus estimates, for ordinary people can not empathize with such acts in any circumstances.

Secondly, supporting the assumption that a perceiver's self-based consensus estimate is generalized to the samplebased consensus estimate, a strong positive correlation was observed between these two types of consensus estimates for all stimulus stories. Thus, as implied in the formulation of false consensus bias, a tendency of the subjects to see their own behavioral choices as common and normative was clearly indicated in the present results. In other words, the conception of the ordinary person was shown to covary with the perceiver's self-conception. As to the relative efficacy of self-based versus sample-based consensus estimates as determinants of responsibility attribution, the results of path analyses indicated that, though not unequivocal, the direct effect of the former tended to be greater than that of the latter. Since 
the greatcr power of self-based consensus was reported by Hansen and Donoghue (1977), the present results may be consistent with their finding, even though, unlike their study, self-based consensus and sample-based consensus were not independently manipulated but self-generated in the present study. At the least, it may be said from the present results that the self-based consensus estimate can affect responsibility attribution independently of its generalization to the samplebased consensus estimate.

Thirdly, the predicted directions of difference between driver and nondriver subjects were confirmed for all relevant dependent measures. Compared to subjects who do not drive, driver subjects generated lower self-based and samplebased consensus estimates regarding the perpetrator's behavior in question and attributed greater responsibility to the perpetrator for the occurrence of the accident. These results are entirely consistent with the prediction that, for automobile accidents, greater responsibility attribution of driver subjects would be mediated by their lowered consensus estimates. In addition, the driver-nondriver difference also emerged for their responses to sanction measures, in that driver subjects were found to be more strict with the perpetrator than were nondriver subjects.

Returning to the issue of self-protective motivational biases, the directions of difference found between driver and nondriver subjects were clearly against Shaver's formulation of blame-avoidance tendency, but in favor of the harmavoidance tendency proposed by Walster. Since an automobile accident is situationally more relevant and a perpetrator (a motorist) personally more similar to drivers than to nondrivers, driver subjects would have assigned lessened responsibility and sanctions to the accident perpetrator had the blame-avoidance tendency prevailed. Thus, the driver-nondriver difference found for their responsibility attribution and sanctioning clearly contradicted the blame-avoidance tendency on the part of driver subjects.

On the other hand, the present results were well in agreement with the harmavoidance tendency, even though the outcome severity of the accident was not manipulated as was typically done to test Walster's proposition. In judging responsibility for an automobile accident, drivers can minimize the threatening implication that they too may be involved in a similar accident by estimating a lowered probability of their reacting as the perpetrator did to the situation in which the accident occurred. Therefore, the lower self-based consensus estimated by driver subjects, to whom the accident is more relevant, can be taken as evidence for their motivational bias of harmavoidance tendency, though it does not preclude other nonmotivational explanations.

Lastly, we should refer to the critiques aimed at responsibility attribution research in general and the formulation of defensive attribution in particular. Provoked by the accumulation of inconsistent findings, especially about the effect of outcome severity on responsibility attribution, these critiques have centered around the meanings of the term responsibility, the abmiguity of the concept being blamed for the confusion in the attribution of responsibility literature. For example, Fishbein and Ajzen (1973) have suggested that responsibility has a different meaning for perceivers making judgments at each of Heider's (1958) five contextual levels of responsibility, and Hart (1968), a legal philosopher, has distinguished different varieties of responsibility such as role, causal, liability, and capacity responsibility. Although psychologists are less concerned with the elucidation of the concept than lawyers and philosophers, a number of researchers have begun to consider qualitative differences in the nature of 
responsibility attributed to others. For example, Shroeder and Linder (1976) have distinguished two aspects of responsibility, causality and culpability, and in a similar vein Fincham and Shultz (1981) have separated the concepts of causation, blame, and restitution. Such conceptual analysis is certainly needed to dispel the confusion in responsibility attribution studies.

In spite of this, the term responsibility was left undefined in the present study for the very simple and practical reason that the subjects did not have such differentiated conceptions of responsibility and the term as used in the present study was clearly understood by them. Instead, the conceptual issue was addressed in different ways. First, the present study incorporated the test-retest procedure to assess the stability of the subjects' responses over time and found the reliability of responsibility judgments to be quite satisfactory. Second, two sanction measures of restitution and punishment were introduced, and the extent of responsibility attributed to the perpetrator was found to be highly correlated with the degree of applicability of sanctions against the perpetrator.

Furthermore, the present study used three different stimulus stories in order to examine the generalizability of the findings obtained. Most of the major results such as correlation patterns observed among the dependent variables and directions of difference found between driver and nondriver subjects for the dependent measures were held intact for all stimulus stories. However, the driver-nondriver difference was not equally manifested for all relevant dependent measures nor for all stimulus stories. As to the dependent measures, the driver-nondriver difference was more clearly pronounced for their consensus estimates than for their responsibility attribution and, of the two sanction measures, applicability of restitution was found to be more sensitive to the dif- ference than that of punishment. More importantly, the degree of the drivernondriver difference was found to be differentially manifested for the different accident situations, even though they had some common structural features. Of the three stimulus stories, the subjects' responses to the accident situation depicted in Case 2 was little affected by the drivernondriver difference. For this stimulus story, the significant driver-nondriver difference was observed only for their selfbased consensus estimates, but it did not generalize to their sample-based consensus estimates nor did it affect their responsibility attribution and sanctioning. Though the clarification of the reasons for the varying manifestations of the drivernondriver difference must await future investigations, these results have some important implications for the conceptual issue of responsibility, since they suggest that the efficacy of an influencing factor would depend not only on how we define responsibility but also on how we construct the stimulus materials to be judged.

\section{References}

Burger, J. M. 1981 Motivational biases in the attribution of responsibility for an accident: $A$ meta-analysis of the defensive-attribution hypothesis. Psychological Bulletin, 90, 496-512.

Chaikin, A. L., \& Darley, J. M. 1973 Victim or perpetrator?: Defensive attribution of responsibility and the need for order and justice. Journal of Personality and Social Psychology, 25, 268-275.

Fincham, F. D., \& Shultz, T. R. 1981 Intervening causation and the mitigation of responsibility for harm. British Journal of Social Psychology, 20, 113-120.

Fishbein, M., \& Ajzen, I. 1973 Attribution of responsibility: A theoretical note. Journal of Experimental Social Psychology, 9, 148-153.

Hagiwara, S., Sono, S., \& Sano, K. 1977 An attributional approach to responsibility judgments for automobile accidents. Keio Studies on Organizational Behavior and Human Performance, 3, 3-39. (In Japanese)

Hansen, R. D., \& Donoghue, J. M. 1977 The power of consensus: Information derived from 
one's own and others' behavior. Joumal of Per. sonality and Social Psychology, 35, 294-302.

Hart, H.L.A. 1968 ptrishment and responsibility: Essays in the philosophy of lawe. New York: Oxford University Press.

Heider, F. 1958 The psychology of interpersonal relations. New York: Wiley.

Kelley, H. H. 1967 Attribution theory in social psychology. In D. Levine (Ed.), Nebraska symposium on motivation, Lincoln: University of Nebraska Press.

Lloyd-Bostock, S. 1979 The ordinary man and the psychology of attributing causes and responsibility. Modem Law Review, 42, 143-168.

McKillip, J., \& Posavac, E.J. 1975 Judgments of responsibility for an accident. Journal of Personality, 43, 248-265.

Phares, E.J., \& Wilson, K.G. 1972 Responsibility attribution: Role of outcome severity, situational ambiguity, and internal-cxternal control. Journal of Personality, 40, 392-406.

Ross, L. 1977 The intuitive psychologist and his shortcomings: Distortions in the attribution process. In L. Berkowitz (Ed.), Advances in experimental social psyctology. Vol. 10. New York: Academic Press.
Schroeder, D. A., \& Linder, D. E. 1976 Effects of actor's causal role, outcome severity, and knowledge of prior accidents upon attributions of responsibility. Journal of Experimental Social $P_{s y-}$ chology, 12, 340-356.

Shaver, K. G. 1970a Defensive attribution: Effects of severity and relevance on the responsibility assigned for an accident. Journal of Personality and Social Psychology, 14, 101-113.

Shaver, K. G. $1970 \mathrm{~b}$ Redress and conscientiousness in the attribution of responsibility for accidents. Journal of Experimental Social Psychology, 6, $100-110$,

Shaw, J. I., \& McMartin, J.A. 1977 Personal and situational determinants of responsibility for an accident. Human Relations, 30, 95-107.

Vidmar, N., \& Crinklaw, L. D. 1974 Attributing responsibility for an accident: A methodological and conceptual critique. Canadian Journal of Behavioral Science, 6, 112-130.

Walster, E. 1966 Assignment of responsibility for an accident. Journal of Personality and Social Psychology, 3, 73-79.

Walster, E. 1967 'Second guessing' important events, fruman Relations, 20, 239-249.

(Received Aug. 30, 1982; accepted March 12, 1983) 